\title{
OPTICAL 3D MEASUREMENTS IN HYDRODYNAMIC TUNNEL FOR AIRCRAFT ICING STUDY
}

\author{
V. A. Knyaz ${ }^{1,2}$, D. G. Stepaniants ${ }^{1}$, E. V. Ippolitov ${ }^{3}$, M. M. Novikov ${ }^{3}$ \\ ${ }^{1}$ State Research Institute of Aviation Systems (GosNIIAS), 125319 Moscow, Russia - knyaz@gosniias.ru \\ ${ }^{2}$ Moscow Institute of Physics and Technology (MIPT), Russia \\ ${ }^{3}$ Institute on Laser and Information Technologies of RAS - \\ Branch Research Centre Crystallography and Photonics of RAS, Shatura, Russia - ippevg@yandex.ru, novikov@ rambler.ru
}

Technical Commission II, WG 10

KEY WORDS: image-based 3D measurements, calibration, accuracy, hydrodynamic tunnel, aircraft icing, multimedia imaging

\begin{abstract}
:
The study of how aircraft icing influences on aircraft aerodynamic performance is very important for developing measures and recommendation to improve aircraft flight safety. The effective method of aerodynamic processes modeling is experiment in wind (aerodynamic) tunnel or water (hydrodynamic) tunnel. They allow to perform experiments with a scaled model of an aircraft affected by icing and to visualize the wing flow process and changes caused by icing. While visualization of the wing flow yields useful qualitative information about flow, it is more important to retrieve quantitative $3 \mathrm{D}$ data of flow, which allows to forecast icing process and to develop anti-icing techniques and recommendations.

The presented study addresses to creating an photogrammetric system and 3D measurement techniques for quantitate evaluation of 3D flow parameters in a hydrodynamic tunnel for aircraft icing influence exploration. Being an initial part of a long-term research project, this study is aimed at developing of an accurate calibration technique of the photogrammetric system for 3D measurement in condition of two optical media interfaces. The developed algorithms for imaging process through two optical media interfaces are used in calibration procedure and object 3D coordinates measuring. The results of the photogrammetric system calibration are given in comparison with standard (single media) case. Experimental 3D reconstruction of a typical object demonstrated high accuracy of the developed algorithms.
\end{abstract}

\section{INTRODUCTION}

The adequate modeling and forecasting of aircraft icing is extremely important for flight safety (Cao et al., 2018), so this process should be studied at the very early stages of an aircraft design. An understanding of ice accretion influence on aircraft aerodynamic characteristics is necessary for develop anti-icing measures. The importance of aircraft icing problem is reflected in a set of international and national standards, according to which the most critical forms of ice accretion, affecting the characteristics and flight qualities of an aircraft, must be determined for each phase of the flight of the aircraft. The results of such special studies can be used to obtain detailed information for choosing the protective equipment and for determining the critical ice formations. This will allow to formulate requirements for antiicing protection and to give recommendations on aircraft protection from icing. Therefore, it is very important to accurately predict the aerodynamic behavior of an aircraft, basing on adequate model of the icing process.

It is very difficult (and mostly impossible) to study icing process in real aircraft flight, so for these purposes aerodynamic or hydrodynamic tunnels are used. They have proved their high efficiency for aerodynamic research due to possibility of monitoring and controlling the flow process. The flow modeling in wind and hydrodynamic tunnels is based on a similitude concept saying that a model adequately reflects real application if geometric, kinematic and dynamic similarity is observed.

${ }^{*}$ Corresponding author
The criterium of results validity in the different fluid mechanics experiments is the equivalence of some dimensionless quantities as Reynolds number, Mach number, Prandtl number, which have to be same in model and real conditions. The advantage of using hydrodynamic tunnel for an aircraft icing study is the possibility of exploration at low flow speed, preserving the same value of the Reynolds numbers $R$, which indicates the ratio of inertial forces to viscous forces within a fluid.

This research is a first stage of a long-term research project for exploring an influence of aircraft icing process on flying characteristics of aircraft by flow modeling in low-speed hydrodynamic tunnel. The main tasks of the research project are: (1) to develop techniques and configuration of optical system for accurate spatial coordinates measurements in multi-media working space hydrodynamic tunnel; (2) to develop techniques for flow registration and accurate 3D reconstruction; (3) to develop techniques of aircraft icing process simulating for modeling in a hydrodynamic tunnel.

The paper presents the results of the first stage of the project related with developing of the imaging model and calibration technique for considered case of 3D measurements through two optic media interfaces and evaluation of the developed algorithms.

\section{RELATED WORK}

Photogrammetric 3D measurement techniques are successfully used in wide variety of applications due to availability of mod- 
ern high-performance imaging sensors and advances in the methods of image processing. In most cases 3D measurements are to perform in standard conditions of single optical media.

Optical measurements based on object image processing are becoming more widespread due to improvements in image acquisition techniques and significant advances in image processing methods. At the same time, the range of applications is expanding, including, among other things, applications that require performing measurements in a workspace that includes several optical media, which affects the process of forming an image of the measured object (Shortis, 2015).

To study 3D characteristic of a flow in a hydrodynamic tunnel requires to carry out optical measurements in multimedia optical working space, including air, glass and liquid (oil or water). The comprehensive review of calibration techniques for 3D measurements by underwater camera systems is given in (Shortis, 2015, Bräuer-Burchardt et al., 2015, Telem and Filin, 2010, Raffel et al., 2018), including analysis of different approaches to system calibration such as estimating distance dependent distortion or applying the special hemispherical dome port for camera (Menna et al., 2016).

An approach for the 3-D mapping of underwater caves (Weidner et al., 2017) utilizes the intersection of the cone of the video-light with the cave boundaries: walls, floor, and ceiling, resulting in the construction of a wire frame outline of the cave. For 3D reconstruction it employs a stereo camera and a video-light. Successive frames are combined using a state of the art visual odometry algorithm while simultaneously inferring scale through the stereo reconstruction. The approach has been experimentally tested at a cave, part of the Sistema Camilo, Quintana Roo, Mexico, resulting in the underwater interior of the cave $3 \mathrm{D}$ reconstruction.

A self-calibrating line laser scanning system (Bleier and Nüchter, 2017) enables the creation of dense 3D models with a single fixed camera and a freely moving hand-held cross line laser projector. The proposed approach for system calibration uses geometric constraints, such as coplanarities for retrieving the depth information. The approach do not use any prior knowledge of the position and orientation of the laser projector. The proposed technique has been studied and compared with 3D reconstruction using explicit calibration, demonstrating applicability as for above-the-water so for underwater scenes 3D scanning..

Three ways for 3D reconstruction has been evaluated for the task of estimating the 3D position of underwater objects (Pedersen et al., 2018). The compared approaches were: an approach relying solely on in-air camera calibration, an approach with the camera calibration performed under water and an approach based on ray tracing with Snell's law. The study has showed that the in-air camera calibration is the most inaccurate as it does not take refraction into account. The precision of the estimated $3 \mathrm{D}$ positions based on the underwater camera calibration and the ray tracing based approach were, on the other hand, almost identical. However, the ray tracing based approach is found to be advantageous as it is far more flexible in terms of the calibration procedure due to the decoupling of the intrinsic and extrinsic camera parameters.

In the field of fluid mechanics, the resolution of computational schemes has outpaced experimental methods and widened the gap between predicted and observed phenomena in fluid flows. Thus, a need exists for an accessible method capable of resolving three-dimensional (3D) data sets for a range of problems.
A technique for performing quantitative 3D imaging of many types of flow fields (Truscott et al., 2013) enables investigation of complicated velocity fields and bubbly flows. A re-parameterization of images captured by an array of cameras by using Light Field Imaging allows to reconstruct a $3 \mathrm{D}$ volumetric map for every time instance, despite partial occlusions in the volume. The technique makes use of an algorithm known as synthetic aperture refocusing, whereby a 3D focal stack is generated by combining images from several cameras post-capture. Light Field Imaging (Belden et al., 2010) allows for the capture of angular as well as spatial information about the light rays, and hence enables 3D scene reconstruction. Quantitative information can then be extracted from the $3 \mathrm{D}$ reconstructions using a variety of processing algorithms. In particular, we have developed measurement methods based on Light Field Imaging for performing 3D particle image velocimetry, extracting bubbles in a 3D field and tracking the boundary of a flickering flame.

Meanwhile the considered research project requires special calibration and 3D measurement techniques for accurate description of imaging process in hydrodynamic tunnel.

\section{HARDWARE AND ALGORITHMS}

\subsection{Hydrodynamic tunnel}

Hydrodynamic tunnel is an effective mean for flow processes investigation in hydrodynamics and aerodynamics. The validity of the modeling results are provided by equivalence of real and modeling Reynolds number, whether a submerged water vehicle model is tested in air or an aerial vehicle is tested in water. Modeling in hydrodynamic tunnel allows to work with very low flow velocity (flow velocity $V=2-10 \mathrm{~cm} / \mathrm{s}$ ). The flow with given speed is formed due to the gravity of the water flowing into the working part from a large container located above. At the same time, the transition from this capacity to the working part is specially organized in the form of a confuser made, and the beginning of the working part is equipped with a honeycomb.

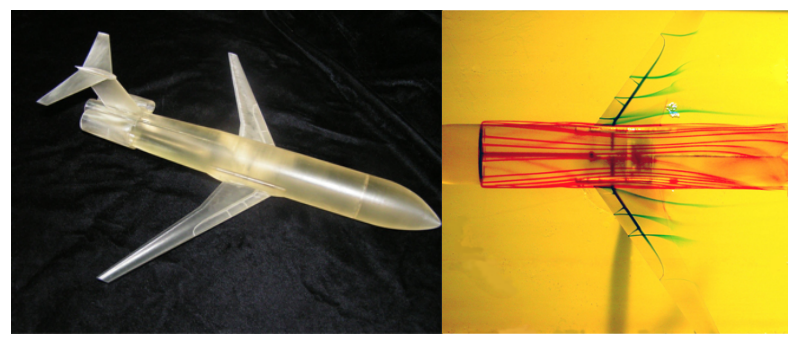

Figure 1. SLA model for flow study and visualization of flows in HDT-400

For study of influence of aircraft icing on flying characteristic a very low-speed hydrodynamic tunnel HDT-400 of The Central Aerohydrodynamic Institute (TsAGI) with a working part size of $400 \times 400 \mathrm{~mm}^{2}$ is used. This hydrodynamic tunnel has been already used for the study of unsteady phenomena in the position of the regions of "explosion" of vortices. Specially designed scaled model of a plane was used for flow visualization (Figure 1). The plane model has special network of inner channels to form colored flow jets for visualization.

\subsection{Optical 3D measurement system}

The registration and 3D measurements of flow characterstics in hydrodynamic tunnel a photogrammetric approach is appied. The 
optical system for 3D measurement is based on original "Mosca" motion capture system (Knyaz, 2015), which software has been extended for multimedia optical measurements.

The scalable 3D motion capture system "Mosca" provides high speed image acquisition and high accuracy of photogrammetric 3D measurements. Depending on the application the system can be easily modified for different working areas from $100 \mathrm{~mm}$ to $10 \mathrm{~m}$. The developed original software supports image acquisition from $2 \ldots 4$ cameras in synchronization mode at frame rate up to 100 frames per second. The Mosca system modified for image acquisition in hydrodynamic tunnel with laboratory setup for calibration technique development and evaluation is shown in Figure 2.

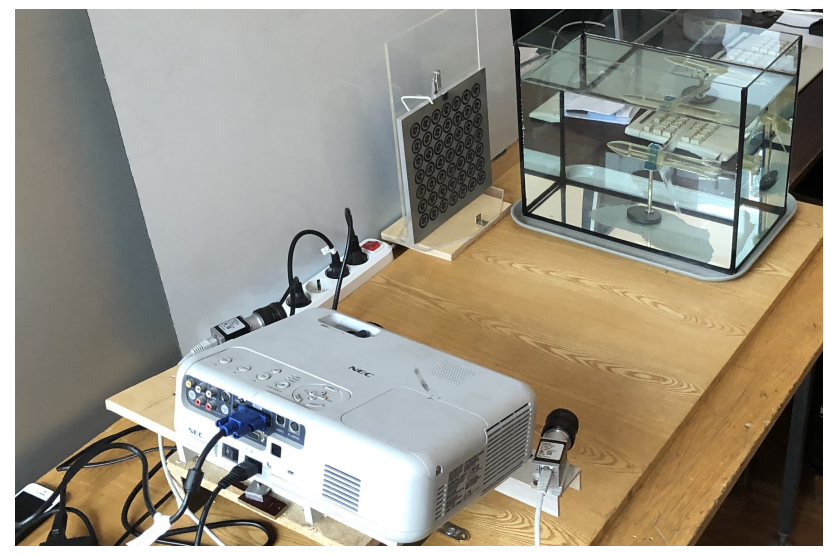

Figure 2. Motion capture system

The developed system calibration for single optical media provides accuracy of $0.01 \%$ of working space. To apply Mosca system for 3D measurements in hydrodynamic tunnel it is necessary to account the phenomenon of light refraction at interfaces of different media. So the original the original techniques for calibration and 3D measurements, based on the detailed analysis of light passing through the different optical media has been developed and evaluated.

\subsection{Single media calibration}

Single media imaging process is based on co-linearity model expressing the condition that the point of the scene $S$, the center of the projection of $C$ and the image of this point $s$ lie on one straight line:

$$
\mathbf{x}_{s}-\mathbf{x}_{p}=-\mu \mathbf{R} \cdot\left(\mathbf{X}_{S}-\mathbf{X}_{C}\right)
$$

Here

$\mathbf{X}_{C}=\left(X_{C}, Y_{C}, Z_{C}\right)^{T}$ - coordinates of the center of the projection,

$\mathbf{X}_{S}=\left(X_{S}, Y_{S}, Z_{S}\right)^{T}-$ coordinates of the scene point $S$,

$\mathbf{x}_{s}=\left(x_{s}, y_{s},-c\right)^{T}-$ the corresponding coordinates of the scene point $S$ in the image,

$\mathbf{R}$ - coordinate system rotation matrix,

$\mathbf{x}_{p}$ - coordinates of the principal point,

$\mu$ - scale factor.

The coordinates of point $S$ and of the center of projection $C$ are defined in object coordinate system $O X Y Z$ (Figure 3), coordinates of image point $\mathbf{x}_{s}$ are defined in image coordinate system Cxyz.
Equations 1 can be written in form:

$$
\begin{aligned}
& x_{s}-x_{p}= \\
& -c \frac{r_{11}\left(X_{S}-X_{C}\right)+r_{12}\left(Y_{S}-Y_{C}\right)+r_{13}\left(Z_{S}-Z_{C}\right)}{r_{31}\left(X_{S}-X_{C}\right)+r_{32}\left(Y_{S}-Y_{C}\right)+r_{33}\left(Z_{S}-Z_{C}\right)} \\
& y_{s}-y_{p}= \\
& -c \frac{r_{21}\left(X_{S}-X_{C}\right)+r_{22}\left(Y_{S}-Y_{C}\right)+r_{23}\left(Z_{S}-Z_{C}\right)}{r_{31}\left(X_{S}-X_{C}\right)+r_{32}\left(Y_{S}-Y_{C}\right)+r_{33}\left(Z_{S}-Z_{C}\right)}
\end{aligned}
$$

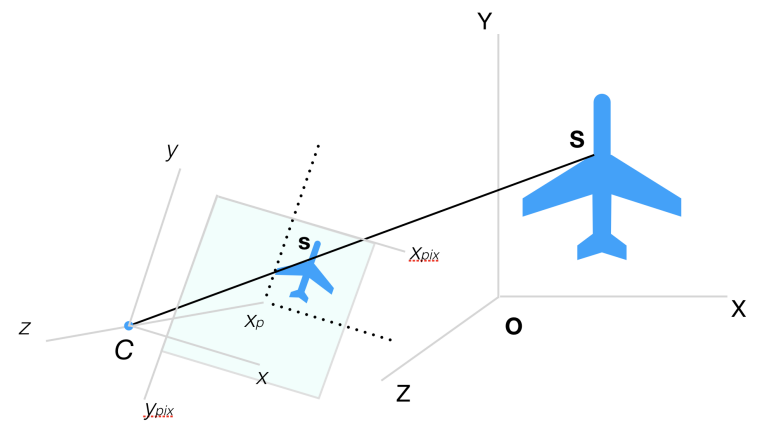

Figure 3. Light ray path for the case of single media

To transform 2D coordinates $\xi_{s}, \eta_{s}$ of the point $S$ in digital image to image coordinates $\mathbf{x}_{s}=\left(x_{s}, y_{s}, z_{s}\right)^{T}$

$$
\begin{aligned}
& x_{s}=m_{x} \cdot\left(\xi_{s}-x_{p}\right) \\
& y_{s}=m_{y} \cdot\left(y_{p}-\eta_{s}\right) \\
& z_{s}=-c .
\end{aligned}
$$

To account for distortions, additional terms are introduced into the collinearity equations $\Delta x$ and $\Delta y$, describing various distorting factors. Then the real (distorted) $\tilde{x}, \tilde{y}$ coordinates of a point in the image are defined as:

$$
\begin{aligned}
& \tilde{x}=x+\Delta x ; \\
& \tilde{y}=y+\Delta y ;
\end{aligned}
$$

The practice of photogrammetric measurements has proved that the good description for nonlinear distortion is the following model (Beyer, 1992):

$$
\begin{aligned}
\Delta x & =a_{0} \cdot y+x\left(a_{1} r^{2}+a_{2} r^{4}+a_{3} r^{6}\right) \\
& +a_{4}\left(r^{2}+2 x^{2}\right)+2 a_{5} x y ; \\
\Delta y & =a_{0} \cdot x+y\left(a_{1} r^{2}+a_{2} r^{4}+a_{3} r^{6}\right) \\
& +a_{5}\left(r^{2}+2 y^{2}\right)+2 a_{4} x y ;
\end{aligned}
$$

with $r^{2}=x^{2}+y^{2}$

Here

$x_{a}, y_{a}$ - coordinates of a point on the image,

$a_{0}, \ldots, a_{5}$ - camera interior orientation parameters:

$a_{0}$ - coefficient of affine distortion;

$a_{1}, a_{2}, a_{3}$ - coefficients of radial distortion;

$a_{4}, a_{5}-$ coefficients of tangential distortion.

The vector $v_{e}^{l}=\left(x_{p}, y_{p}, m_{x}, m_{y}, a_{0}, \ldots, a_{5}\right)^{T}$ of estimated interior orientation parameters for test field calibration includes co- 
ordinates of principal point, image scales and additional parameters correspondingly, spatial coordinates of reference points being known by independent precise measurements.

The unknown parameters are determined by least mean square estimation using image coordinates of a set of flat testfield reference points as observations (Knyaz, 2006, Chibunichev et al., 2019).

\subsection{Imaging of an object behind two media interfaces}

In case of image acquisition of an object located in hydrodynamic tunnel, light ray from an object point $S$ projected into the image point $s$ passes through optical media with differing refractive indices, crossing two media interfaces.

Ray path from an object point $S$ imaged by camera as image point $s$ is shown in Figure 4. To provide accurate 3D measurements it is necessary to take into account light refraction at two media interfaces: "air-glass" and "glass-liquid (water)".

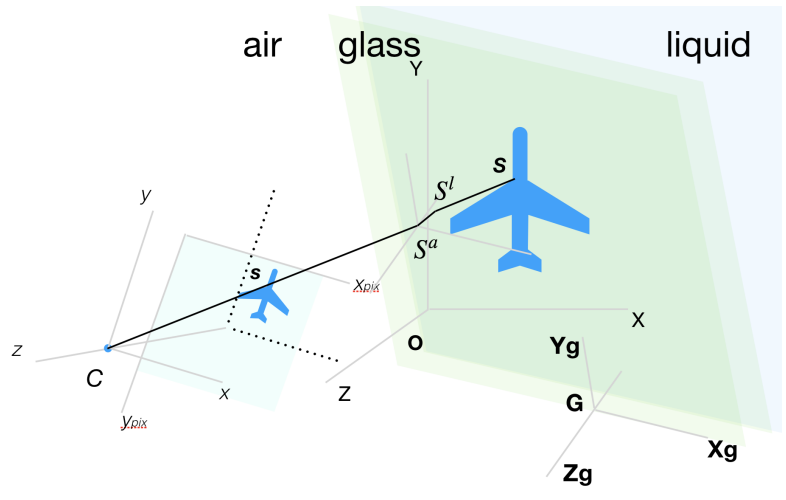

Figure 4. Light ray path for the case of two media interfaces

The ray path for an image point $s$ in this case contains three segments defined by vectors $\vec{l}, \vec{g}, \vec{a}$ determining direction of the ray in liquid, glass and air correspondingly.

Vector $\vec{a}$ having image coordinates $\left(x_{s}, y_{s},-c\right)^{T}$ has $\left(a_{x}, a_{y}, a_{z}\right)^{T}$ in object coordinate system defined as:

$$
\vec{a}=\left(\begin{array}{l}
a_{x} \\
a_{y} \\
a_{z}
\end{array}\right)=\mathbf{R} \cdot\left(\begin{array}{c}
x_{s} \\
y_{s} \\
-c
\end{array}\right)
$$

Let glass coordinates system $G X_{g} Y_{g} Z_{g}$ is defined in the object coordinate system by its origin point $G$ and by rotation matrix $M_{g} . X_{g}$ axis and $Y_{g}$ axis lie in the plane of the glass surface and $Z_{g}$ axis is perpendicular to the glass surface (Figure 4). To find full ray path for the image point $s$ it is necessary to find the point $S^{a}$ of intersection of vector $\vec{a}$ and the frontal glass surface $X_{g} Y_{g}$ and the point $S^{l}$ of intersection $\vec{l}$ and the rear glass surface $X_{g}^{\prime} Y_{g}^{\prime}$.

The coordinates of projection center $C$ in glass coordinate system is defined by:

$$
\begin{array}{r}
\boldsymbol{C}_{g}=\mathbf{M}_{g} \cdot(\boldsymbol{C}-\boldsymbol{G}) \\
\vec{a}_{g}=\mathbf{M}_{g} \cdot \vec{a}
\end{array}
$$

The coordinates of the point $S^{a}$ in the glass coordinate system are found from the condition of $Z_{S_{g}}=0$ :

$$
\boldsymbol{S}_{g}^{a}=\mathbf{C}_{g}-\frac{Z_{C g}}{z_{a}} \cdot \vec{a}_{g}
$$

The direction of vector $\vec{g}$ can be found as:

$$
\vec{g}=\left(\begin{array}{c}
g_{x} \\
g_{y} \\
g_{z}
\end{array}\right)=\left(\begin{array}{c}
a_{x} \cdot \operatorname{tg}\left(\theta_{g}\right) \\
a_{y} \cdot \operatorname{tg}\left(\theta_{g}\right) \\
a_{z}
\end{array}\right)
$$

with

$$
\sin \left(\theta_{g}\right)=\frac{1}{n_{g}} \cdot \sin \left(\theta_{a}\right)
$$

Similarly for "glass-liquid" interface

$$
\overrightarrow{l_{g}}=\left(\begin{array}{c}
l_{x} \\
l_{y} \\
l_{z}
\end{array}\right)=\left(\begin{array}{c}
g_{x} \cdot t g\left(\theta_{l}\right) \\
g_{y} \cdot t g\left(\theta_{l}\right) \\
g_{z}
\end{array}\right)
$$

with

$$
\begin{aligned}
\sin \left(\theta_{l}\right) & =\frac{n_{g}}{n_{l}} \cdot \sin \left(\theta_{g}\right) . \\
\boldsymbol{S}_{g}^{l} & =\mathbf{S}_{g}^{a}+\vec{g}_{g}
\end{aligned}
$$

Transformation into the object coordinate system give the following equations:

$$
\begin{array}{r}
\boldsymbol{S}^{l}=\mathbf{M}_{g}^{T} \cdot \boldsymbol{S}_{g}^{l} \\
\vec{l}=\mathbf{M}_{g}^{T} \cdot \vec{l}_{g}
\end{array}
$$

Equations (8) - (16) allow to find for any image point $s$ the light ray in liquid $\vec{l}$ to corresponding object point $S$, which are needed for camera calibration and object 3D coordinates determination.

\section{RESULTS AND DISCUSSION}

The developed algorithms has been evaluated for laboratory setup shown in Figure 2. The photogrammetric 3D measurement system includes two Basler acA1280-60gm GigE cameras with the e2v EV76C560 CMOS sensor and NEC multi media projector mounted on a rigid platform, providing stable exterior orientation. The main characteristics of Basler acA1280-60gm GigE camera are presented in Table 1.

\begin{tabular}{lr}
\hline \hline Parameter & Value \\
\hline Sensor Type & CMOS \\
Sensor Size, mm & $6.8 \times 5.4$ \\
Resolution (HxV), pix & $1282 \times 1026$ \\
Resolution, Mpix & 1.3 \\
Pixel Size (H x V), $\mu m$ & $5.3 \times 5.3$ \\
Frame Rate, fps & 60 \\
Interface & GigE \\
Pixel Bit Depth, bits & 12 \\
\hline \hline
\end{tabular}

Table 1. Main characteristics of Basler acA1280-60gm GigE camera

For estimating the quality of system calibration using the developed algorithms calibration procedure has been carried out as for single media (air) so for two media interfaces imaging. 


\begin{tabular}{l|rr|rr}
\hline \hline Parameter & \multicolumn{2}{|c|}{ Left camera } & \multicolumn{2}{c}{ Right camera } \\
& single media case & multi media case & single media case & multi media case \\
\hline$\sigma_{c e}, m m$ & 0.045 & 0.045 & 0.045 & 0.045 \\
\hline$m_{x}, m m$ & 0.00513 & 0.00510 & 0.00512 & 0.00510 \\
$m_{y}, m m$ & 0.00514 & 0.00510 & 0.00512 & 0.00510 \\
$b_{x}, p i x$ & 633 & 636 & 650 & 647 \\
$b_{y}, p i x$ & 514 & 512 & 498 & 494 \\
\hline \multicolumn{4}{|c}{ Distortion } \\
\hline$a_{0}$ & 0.00039 & 0.00043 & -0.00141 & -0.000750 \\
$a_{1}$ & -0.000909 & -0.000870 & -0.000768 & -0.000769 \\
$a_{2}$ & 0.000164 & 0.000213 & 0.000208 & 0.000234 \\
$a_{3}$ & 0.000531 & 0.000478 & 0.000527 & 0.000509 \\
$a_{4}$ & -0.000239 & -0.000150 & 0.0000989 & 0.0000485 \\
$a_{5}$ & 0.000569 & 0.000450 & 0.000295 & 0.000237 \\
\hline \hline
\end{tabular}

Table 2. Calibration results

For calibration a flat test field (Figure 5) with 49 reference points marked by coded targets (Knyaz and Maksimov, 2014) has beed used. Fifteen images of the test field for each camera were acquired at different test field positions and orientation to the cameras, with the first test field position determining the object coordinate system.

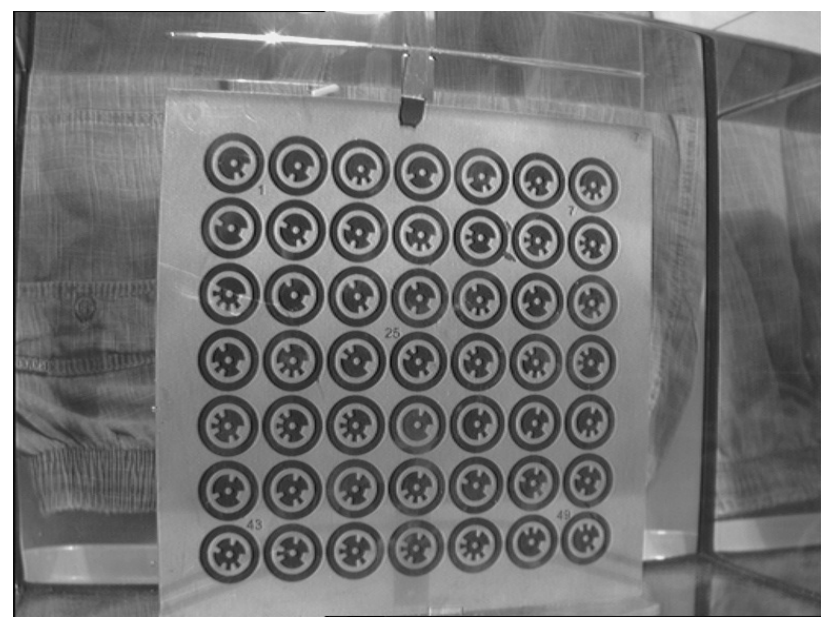

Figure 5. A calibration image of test field in a liquid

The developed calibration procedure determines the following parameters: interior orientation parameters, external orientation parameters, positions and orientations of test field for all calibration images and the coordinates of the test field reference points in local test field coordinate system.

The calibration results of the complex are given in Table 2 comparison with the calibration results in the absence of glass and an aqueous medium.

Also the results of calibration were evaluated by 3D reconstruction by structured light technique of some typical surface (3D model of aircraft) placed in a liquid, with further comparing the reconstructed surface with accurate reference surface Figure 6.

Figure 6 demonstrates high degree of correspondence between the $3 \mathrm{D}$ model reconstructed using multi media imaging algorithms and the reference 3D model. The mean difference between two surfaces is at the level of $0.01 \mathrm{~mm}$ for the object of about $200 \mathrm{~mm}$.

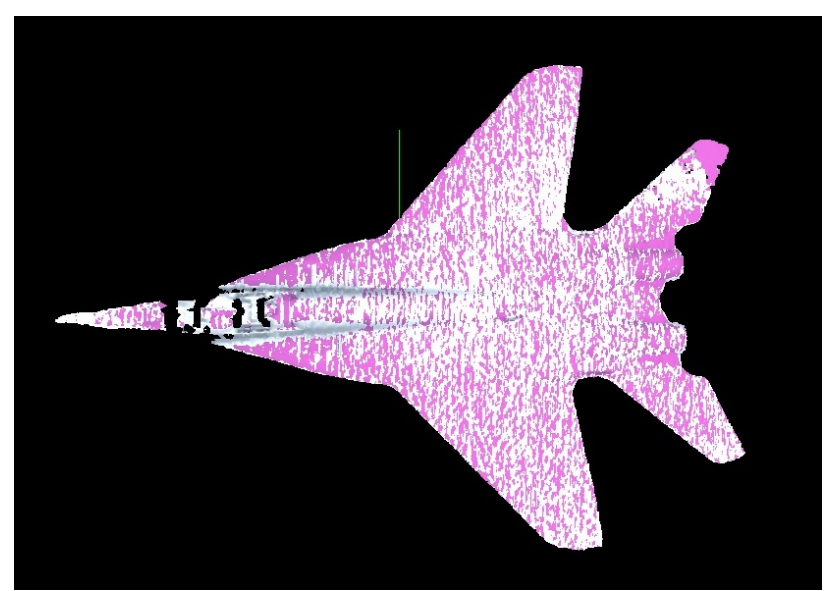

Figure 6. Comparison of the reconstructed surface with accurate reference surface

The table 3 shows the results of evaluating the angular parameters of the external orientation of cameras obtained by calibration in a homogeneous optical medium and in the presence of optical media interfaces.

\begin{tabular}{lcc}
\hline \hline Parameter & \multicolumn{2}{c}{ Calibration } \\
& one-media & multi-media \\
\hline$\alpha_{0}$ & 18.08 & 17.92 \\
$\kappa_{0}$ & -0.32 & -0.28 \\
$\alpha_{1}$ & -18.4 & -18.2 \\
$\omega_{1}$ & -0.39 & -0.36 \\
$\kappa_{1}$ & 0.39 & 0.33 \\
\hline
\end{tabular}

Table 3. Relative orientation

The results of the system cameras calibration for image acquisition through two media interfaces were compared with the system calibration results for single media image acquisition. The parameters estimates for three media case differ from corresponding parameters for single media case by less then $3 \%$.

The comparison demonstrated high accuracy of 3D measurements in water media at the level of $0.02 \mathrm{~mm}$ for working space of $300 \times 300 \times 300 \mathrm{~mm}^{3}$. 


\section{CONCLUSION}

The algorithms of object imaging through two media interfaces is developed for optical 3D measurements in hydrodynamic tunnel for icing process study. The algorithms define the ray path through two media interfaces for every image point providing the basis for accurate 3D measurements in a hydrodynamic tunnel.

The evaluation of the developed algorithms for calibration of the photogrammetric 3D measurement system and for 3D measurements by this system demonstrated high accuracy of system calibration and 3D measurements. The developed technique for photogrammetric system calibration and 3D measurements demonstrated applicability for the task of 3D flow analysis in hydrodynamic tunnel.

\section{ACKNOWLEDGEMENTS}

The reported study was funded by Russian Foundation for Basic Research (RFBR) according to the research project 19-29-13040.

\section{REFERENCES}

Belden, J., Truscott, T., Axiak, M. and Techet, A., 2010. Three-dimensional synthetic aperture particle imaging velocimetry. Measurement Science and Technology 21(12), pp. 125403.

Beyer, H., 1992. Advances in characterization and calibration of digital imaging systems. Int. Arch. Photogramm. Remote Sens. Spatial Inf. Sci. XXIX, pp. 545-555.

Bleier, M. and Nüchter, A., 2017. Low-cost 3d laser scanning in air orwater using self-calibrating structured light. ISPRS - International Archives of the Photogrammetry, Remote Sensing and Spatial Information Sciences XLII-2/W3, pp. 105-112.

Bräuer-Burchardt, C., Kühmstedt, P. and Notni, G., 2015. Combination of air- and water-calibration for a fringe projection based underwater 3d-scanner. In: G. Azzopardi and N. Petkov (eds), Computer Analysis of Images and Patterns, Springer International Publishing, Cham, pp. 49-60.

Cao, Y., Tan, W. and Wu, Z., 2018. Aircraft icing: An ongoing threat to aviation safety. Aerospace Science and Technology 75, pp. $353-385$.

Chibunichev, A. G., Govorov, A. V. and Chernyshev, V. E., 2019. Research of the camera calibration using series of images with common center of projection. ISPRS - International Archives of the Photogrammetry, Remote Sensing and Spatial Information Sciences XLII-2/W18, pp. 19-22.

Knyaz, V. A., 2006. Automated calibration technique for photogrammetric system based on a multi-media projector and a ccd camera. ISPRS - International Archives of the Photogrammetry, Remote Sensing and Spatial Information Sciences XXXVI-5, pp. 1-5.

Knyaz, V. A., 2015. Scalable photogrammetric motion capture system "mosca": Development and application. ISPRS - International Archives of the Photogrammetry, Remote Sensing and Spatial Information Sciences XL-5/W6, pp. 43-49.

Knyaz, V. A. and Maksimov, A. A., 2014. Photogrammetric technique for timber stack volume contol. ISPRS - International Archives of the Photogrammetry, Remote Sensing and Spatial Information Sciences XL-3, pp. 157-162.

Menna, F., Nocerino, E., Fassi, F. and Remondino, F., 2016. Geometric and optic characterization of a hemispherical dome port for underwater photogrammetry. Sensors.
Pedersen, M., Bengtson, S. H., Gade, R., Madsen, N. and Moeslund, T. B., 2018. Camera calibration for underwater $3 \mathrm{~d}$ reconstruction based on ray tracing using snell's law. In: 2018 IEEE/CVF Conference on Computer Vision and Pattern Recognition Workshops (CVPRW), pp. 1491-14917.

Raffel, M., Willert, C. E., Scarano, F., Kähler, C. J., Wereley, S. T. and Kompenhans, J., 2018. Stereoscopic PIV. Springer International Publishing, Cham, pp. 285-307.

Shortis, M., 2015. Calibration techniques for accurate measurements by underwater camera systems. Sensors 15(12), pp. 30810-30826.

Telem, G. and Filin, S., 2010. Photogrammetric modeling of underwater environments. ISPRS Journal of Photogrammetry and Remote Sensing 65(5), pp. 433 - 444.

Truscott, T., Belden, J., Nielson, J., Daily, D. and Thomson, S., 2013. Determining 3d flow fields via multi-camera light field imaging. Journal of Visualized Experiments 73(e4325), pp. 1-9.

Weidner, N., Rahman, S., Li, A. Q. and Rekleitis, I., 2017. Underwater cave mapping using stereo vision. In: 2017 IEEE International Conference on Robotics and Automation (ICRA), pp. 5709-5715. 